\title{
ÉDITORIAL
}

\section{Des échanges fructueux}

Notre très actif correspondant suédois, Lars Persson, a invité en juin dernier les responsables de publications traitant de radioprotection. Plusieurs types de publications étaient représentés : les journaux solidement ancrés au hit parade des meilleures revues, telle Radiation Protection Dosimetry ; des journaux représentant des sociétés nationales de radioprotection, Radioprotection bien évidemment; mais aussi nos collègues anglais, Journal of Radiological Protection, germanophones, Strahlenschutzpraxis; et enfin des lettres, telles SSI newsletter et Alara newsletter. Nous étions douze au total. L'accueil à la suédoise a été chaleureux, un sympathique dîner, accompagné d'un exposé plein d'humour de Bo Lindell, ancien président de la CIPR, sur l'histoire de cette commission, a clôturé le tout.

Les discussions ont été fructueuses et de celles-ci est ressorti un ostracisme criant de la part des officines chargées d'indexer nos écrits, nous sommes tout simplement « snobés» par la plus grande d'entre elle. Radiation Protection Dosimetry que tout le monde considère comme un excellent journal n'est pas cité, Health Physics, qui reste une référence, est à la limite de l'exclusion. Comment réagir, telle a été la question, mais la solution n'est pas simple car nos activités sont appliquées ce qui n'est pas bien vu dans ce monde de sélection. Ceci a une influence sur notre revue, puisque l'une des conditions pour être indexé est de publier 6 numéros par an, mais chambouler notre système pour un tel résultat ne sert à rien. Vérifions bien si nous sommes indexés sur les bases du nucléaire et faisons des efforts par ailleurs.

Ceci doit-il nous décourager et nous conduire à publier n'importe quoi ? La question ne se pose pour nul d'entre nous, et nous avons tous mis en avant notre système de lecteurs anonymes garant de la qualité des articles, y compris pour la publication des actes des colloques. Radioprotection-colloques qui veut rivaliser avec les numéros spéciaux de Radiation Protection Dosimetry n'y déroge pas. Ceci n'est pas sans difficulté et le professeur Klaus Becker, ancien éditeur de la revue allemande dont les positions sont toujours stimulantes, n'a pas manqué de signaler les difficultés de l'exercice et il a accepté de nous faire part de ses réflexions sous notre rubrique désormais active «lettre à la rédaction». Il nous faut bien réfléchir aux questions qu'il pose et que nous nous sommes posées si nous voulons satisfaire nos lecteurs. Je peux vous garantir qu'au comité de rédaction de la revue, représentant bien des courants de pensée, nous nous y efforçons. Réussissons-nous? C'est à vous de nous le dire, mais vos réponses à notre dernière enquête étaient rassurantes.

Alors quoi de neuf pour tenter de répondre positivement à ces interrogations ? Déjà, notons que notre journal s'ouvre au-delà de nos frontières et c'est avec plaisir que je salue la venue d'un article pakistanais. Nos évaluateurs, vous l'avez sûrement 
remarqué, viennent de tous horizons et de pays de plus en plus divers. Enfin nous avons décidé lors d'une dernière réunion du comité de rédaction d'inciter nos collègues français à écrire les articles fondamentaux en anglais, gage de la percée de notre journal à l'étranger. La réunion de Stockholm l'a montré, l'anglais est de plus en plus incontournable. Le Web le montre aussi, plus de 1000 consultations par numéro chez EDP Sciences, mais qui ne vont guère au-delà de la table des matières lorsqu'il est notifié que les articles sont en français. Mais rassurez-vous, l'équilibre entre les deux langues perdurera, nous y veillerons

Le comité de rédaction continue ses réunions délocalisées, depuis 7 ans et après Golfech, Limoges, Brest, Cadarache, Malvesi, nous avons tenu une réunion de notre comité à Brennilis au cœur d'une opération de démantèlement, oh ! pardon, une opération de déconstruction. Nous n'avons pas rencontré l'Ankou' mais nous fûmes accueillis de manière très chaleureuse et nous avons constaté que la radioprotection est bien vivante sur le chantier, un article dans un prochain numéro en témoignera. L'un de nos membres qui avait participé à l'élaboration de cette centrale était bien nostalgique, nul doute que cette nouvelle histoire sera débattue dans le «club histoire».

Alors continuons à faire de Radioprotection une revue lue et appréciée, ceci dépend principalement de vous.

À très bientôt,

H. MÉTIVIER

Président du comité de la revue

\section{Erratum}

Dans le précédent numéro, Christian Lefaure introduisait, dans son éditorial, la traduction d'une étude de cas britannique qui, pour des raisons techniques, ne paraît que dans le présent numéro. Nous nous excusons de cette publication différée.

${ }^{1}$ Relisez Spirou et Fantasio, mais rassurez-vous les personnages que nous avons rencontrés sont bien plus sympathiques et sérieux. 